Classification

Physics Abstracts

$03.65-03.65 \mathrm{~N}$

\title{
Least-bias description of atomic beams
}

\author{
Berthold-Georg Englert (*), Christian Miniatura and Jacques Baudon \\ Laboratoire de Physique des Lasers, Institut Galilée, Université Paris-Nord, Avenue J.B. \\ Clément, 93430 Villetaneuse, France
}

(Received 8 April 1994, accepted in final form 25 July 1994)

\begin{abstract}
We show how an unbiased quantal description of the center-of-mass properties of an atomic beam is achieved by employing an appropriate statistical operator. A few examples with experimental relevance are treated in order to illustrate the method. We find that the quantum corrections to the classical relation between the velocity distribution and the time-offlight signal are negligible in reasonable experimental situations. We apply the method to atoms traversing a longitudinal Stern-Gerlach interferometer where they are exposed to a magnetic field gradient for a short period. The dependence of the fringe visibility upon the strength and duration of the gradient is derived.
\end{abstract}

\section{Introduction.}

In atomic beam experiments the center-of-mass (CM) motion of the atoms is usually not of any particular interest in itself. It merely serves the purpose of getting the atom first into and then out of a region where the internal atomic degrees of freedom are manipulated. The signal recorded refers to this internal structure; as a rule, it does not reveal details of the CM motion. Therefore, one is not led astray when describing the CM motion in classical terms, as if the atom had a well-defined position and velocity at all times.

Recently, however, with the advent of atom interferometry, quantal aspects of the CM motion have become important. In theoretical treatments, it is common to represent the $\mathrm{CM}$ state by a Schrödinger wave function. This may be, and frequently is, a rather bold idealization if the actual information at hand is limited and does not specify a pure CM state uniquely. A more appropriate approach employs a statistical or density operator, rather than a pure-state wave function. This statistical operator has to account for what is known in an unbiased fashion, that is: without any additional hidden assumptions. It is the purpose of this paper to give explicit examples for such statistical operators.

(*) Permanent address: Sektion Physik, Universität München, Am Coulombwall 1, D-85748 Garching, Germany; also at Max-Planck-Institut für Quantenoptik, Hans-Kopfermann-Strasse 1, D-85748 Garching, Germany. 
Here is a brief outline. In the next section we formulate the general strategy, which is based upon maximizing the entropy, subject to the constraints set by the actual knowledge. Then in section 3.2 we illustrate the procedure for a few exemplary experimental situations. One ingredient is always the position known, within limits, at the initial time. In our examples it is supplemented by some knowledge about the velocity distribution, or about a final position ("passive velocity selection"), or about a time-of-flight distribution. The relation between the velocity profile and the time-of-flight signal is then discussed. As an application we consider a longitudinal Stern-Gerlach interferometer with a pulsed magnetic field gradient. We close with a summary.

\section{General strategy.}

2.1 Statistical OPERATOR. - For simplicity we restrict ourselves to one-dimensional motion along the $x$ axis. The formalism is easily extended to three dimensions if necessary. The CM degree of freedom is described by the position and momentum operators $x(t)$ and $p(t)$ with eigenstates $\left|x^{\prime}, t\right\rangle$ and $\left|p^{\prime}, t\right\rangle$,

$$
\begin{aligned}
& x(t)\left|x^{\prime}, t\right\rangle=\left|x^{\prime}, t\right\rangle x^{\prime}, \\
& p(t)\left|p^{\prime}, t\right\rangle=\left|p^{\prime}, t\right\rangle p^{\prime} .
\end{aligned}
$$

Equivalent numerical representations of the statistical operator

$$
\rho(t) \equiv \rho(t, x(t), p(t))
$$

are its $x$ and $p$ matrices

$$
\begin{aligned}
\rho_{\mathrm{x}}\left(t, x^{\prime}, x^{\prime \prime}\right) & =\left\langle x^{\prime}, t|\rho(t)| x^{\prime \prime}, t\right\rangle, \\
\rho_{\mathrm{p}}\left(t, p^{\prime}, p^{\prime \prime}\right) & =\left\langle p^{\prime}, t|\rho(t)| p^{\prime \prime}, t\right\rangle,
\end{aligned}
$$

as well as its Wigner function [1]

$$
\begin{aligned}
\rho_{\mathrm{w}}\left(t, x^{\prime}, p^{\prime}\right) & =\int \mathrm{d} s \rho_{\mathrm{x}}\left(t, x^{\prime}-s / 2, x^{\prime}+s / 2\right) \exp \left(i s p^{\prime} / \hbar\right) \\
& =\int \mathrm{d} q \rho_{\mathrm{p}}\left(t, p^{\prime}+q / 2, p^{\prime}-q / 2\right) \exp \left(i x^{\prime} q / \hbar\right) .
\end{aligned}
$$

For our purposes, it is more convenient to use $\rho_{\mathrm{w}}$ than $\rho_{\mathrm{x}}$ or $\rho_{\mathrm{p}}$. The latter functions are obtained by Fourier transformations as soon as $\rho_{\mathrm{w}}\left(t, x^{\prime}, p^{\prime}\right)$ is known.

The knowledge that we could have about $\rho$ consists of known expectation values $a_{1}, ., a_{n}$ of certain observables $A_{1}, ., A_{n}$ :

$$
\left\langle A_{\jmath}\right\rangle=\operatorname{tr}\left\{A_{\jmath} \rho\right\}=a_{\jmath}
$$

for $j=1,2, ., n$. The least-bias description, consistent with this knowledge, is given by that statistical operator $\rho$ which maximizes the entropy

$$
\eta=-\operatorname{tr}\{\rho \log \rho\},
$$

subject to the constraints (2.5), which are supplemented by the requirement that $\rho$ is normalized to unit trace. In close analogy to classical statistical physics this challenge is met by [2]

$$
\rho=\frac{Z}{\operatorname{tr}\{Z\}}
$$


with the "partition operator"

$$
Z=\exp \left(-\alpha_{1} A_{1}-\alpha_{2} A_{2}-\cdot-\alpha_{n} A_{n}\right) .
$$

The operators $A_{1}, ., A_{n}$ are, of course, functions of the position $x$ and the momentum $p$, and the numerical parameters $\alpha_{1}, ., \alpha_{n}$ are Lagrange multipliers. Their values are determined by

$$
a_{\jmath}=\left\langle A_{\jmath}\right\rangle=-\frac{\partial}{\partial \alpha_{\jmath}} \log (\operatorname{tr}\{Z\})
$$

for $j=1, . \quad, n$. Although we have written these fundamental equations for a finite number of constraints, it is clear that $j$ can just as well symbolize a continuous label. The sum in (2.8) is then replaced by an integral, and the partial derivative in (2.9) by a functional one.

The trace of $Z$, needed in (2.7) and (2.9), can be evaluated by integrating the Wigner function $Z_{\mathrm{w}}\left(x^{\prime}, p^{\prime}\right)$ that is associated with the operator $Z$ just like $\rho_{\mathrm{w}}$ with $\rho$ in (2.4),

$$
\operatorname{tr}\{Z\}=\int \frac{\mathrm{d} x^{\prime} \mathrm{d} p^{\prime}}{2 \pi \hbar} Z_{\mathrm{w}}\left(x^{\prime}, p^{\prime}\right)
$$

In all the examples discussed in the next section, the operators $A_{1}, \ldots, A_{n}$ possess rather simple Wigner functions, so that the Wigner function $(\log Z)_{\mathrm{w}}$ is immediately available. Then the semiclassical approximation

$$
Z_{\mathrm{w}} \cong \exp \left((\log Z)_{\mathrm{w}}\right)
$$

can be employed. As pointed out by Wigner in his seminal paper of 1932 [3] it neglects contributions whose relative size is measured by the squared ratio of a quantal and a classical action. In the present context of atomic-beam kinematics, equation (2.11) is, therefore, expected to be well justified. Consequently, the approximation

$$
\operatorname{tr}\{Z\} \cong \int \frac{\mathrm{d} x^{\prime} \mathrm{d} p^{\prime}}{2 \pi \hbar} \exp \left((\log Z)_{\mathrm{w}}\left(x^{\prime}, p^{\prime}\right)\right)
$$

and also the corresponding one for $\rho_{w}$ obtained by combining (2.11) and (2.12), will work quite well. A specific example that demonstrates the accuracy of $(2.11)$ is given below in section 3.2

It may be worth mentioning that the approximation (2.11) can be used for a simple, direct derivation of the WKB quantization rule [4], a derivation that makes no use of approximate WKB wave functions. This confirms that the approximation (2.11) is semiclassical in nature [5].

2.2 Temporal evolution. - Since $\rho$ is the statistical operator, it does not change in time as a whole,

$$
\frac{\mathrm{d}}{\mathrm{d} t} \rho(t, x(t), p(t))=0 \text {. }
$$

Heisenberg's equation of motion reads here

$$
\frac{\partial}{\partial t} \rho+\frac{i}{\hbar}[H, \rho]=0
$$

it determines the explicit $t$ dependence of $\rho$. A priori, both $\rho$ and the Hamilton operator $H$ are regarded as functions of $t, x(t)$, and $p(t)$; inasmuch as only the explicit $t$ dependence of $\rho$ matters in (2.14), however, it is permissible to replace $x(t)$ and $p(t)$ by $x\left(t^{\prime}\right)$ and $p\left(t^{\prime}\right)$ as long as they refer to any common time. Consequently, the equation of motion (2.14) obeyed by $\rho$ - this special case of Heisenberg's equation of motion is usually called "von Neumann equation" - is equally valid in the "Schrödinger picture" as in the "Heisenberg picture"; indeed, to the extent 
that "pictures" are related to each other through unitary transformations, equation (2.14) is picture independent.

As a consequence of $\mathrm{d} \rho / \mathrm{d} t=0$, the changes from the dynamical evolution of $x(t)$ and $p(t)$ are compensated for by the explicit time dependence of $\rho$. As soon as the functional dependence of $\rho$ upon $x$ and $p$ is known at some reference time $\bar{t}$, the identity

$$
\rho(t, x(t), p(t))=\rho(\bar{t}, x(\bar{t}), p(\bar{t}))
$$

supplies the statistical operator at any other time $t$. This requires that one solves the equations of motion

$$
\begin{aligned}
\frac{\mathrm{d}}{\mathrm{d} t} x & =\frac{i}{\hbar}[H, x], \\
\frac{\mathrm{d}}{\mathrm{d} t} p & =\frac{i}{\hbar}[H, p],
\end{aligned}
$$

and expresses $x(\bar{t})$ and $p(\bar{t})$ in terms of $x(t)$ and $p(t)$. As an example, consider a time dependent net force $F(t)$ acting on an atom with mass $m$. Then

$$
p(t)=p(\bar{t})+\int_{\bar{t}}^{t} \mathrm{~d} t^{\prime} F\left(t^{\prime}\right)
$$

and

$$
x(t)=x(\bar{t})+p(\bar{t}) \frac{t-\bar{t}}{m}+\int_{\bar{t}}^{t} \mathrm{~d} t^{\prime} \frac{t-t^{\prime}}{m} F\left(t^{\prime}\right)
$$

imply

$$
\rho(t, x, p)=\rho\left(\bar{t}, x-p \frac{t-\bar{t}}{m}+\int_{\bar{t}}^{t} \mathrm{~d} t^{\prime} \frac{t^{\prime}-\bar{t}}{m} F\left(t^{\prime}\right), p-\int_{\bar{t}}^{t} \mathrm{~d} t^{\prime} F\left(t^{\prime}\right)\right) .
$$

The same linear transformation connects the corresponding Wigner functions in this simple dynamical situation.

\section{Examples.}

3.1 Simplest EXAMPLE. - In the simplest situation we know the average values $x_{0}$ and $p_{0}$ of the position $x(t)$ and the momentum $p(t)$ as well as their spreads $\delta x_{0}$ and $\delta p_{0}$ at the initial time. This example has been worked out repeatedly; see, for example, references [6] and [7].

Upon suppressing the time argument, the constraints (2.5) are here

$$
\begin{gathered}
\langle x\rangle=x_{0},\left\langle x^{2}\right\rangle=x_{0}^{2}+\left(\delta x_{0}\right)^{2}, \\
\langle p\rangle=p_{0},\left\langle p^{2}\right\rangle=p_{0}^{2}+\left(\delta p_{0}\right)^{2}
\end{gathered}
$$

The partition operator $(2.8)$ is conveniently written in the form

$$
Z=\exp \left(-\kappa^{2}(x-a)^{2}-\lambda^{2}(p-b)^{2}\right)
$$

so that the Lagrange multipliers $\kappa^{2}, \lambda^{2}, a, b$ (with $\kappa>0$ and $\lambda>0$ ) are determined by

$$
\begin{gathered}
\frac{\partial}{\partial a} \log (\operatorname{tr}\{Z\})=2 \kappa^{2}\langle x-a\rangle=2 \kappa^{2}\left(x_{0}-a\right), \\
\frac{\partial}{\partial b} \log (\operatorname{tr}\{Z\})=2 \lambda^{2}(p-b\rangle=2 \lambda^{2}\left(p_{0}-b\right),
\end{gathered}
$$


and

$$
\begin{gathered}
-\frac{\partial}{\partial \kappa^{2}} \log (\operatorname{tr}\{Z\})=\left\langle(x-a)^{2}\right\rangle=\left(x_{0}-a\right)^{2}+\left(\delta x_{0}\right)^{2} \\
-\frac{\partial}{\partial \lambda^{2}} \log (\operatorname{tr}\{Z\})=\left\langle(p-b)^{2}\right\rangle=\left(p_{0}-b\right)^{2}+\left(\delta p_{0}\right)^{2}
\end{gathered}
$$

The Gaussian operator (3.2) is essentially the partition operator of a harmonic oscillator at some temperature, and therefore its trace

$$
\operatorname{tr}\{Z\}=\frac{1}{2 \sinh (\hbar \kappa \lambda)}
$$

is evaluated easily. Then equations (3.3) imply

$$
a=x_{0}, b=p_{0}, \kappa^{2}=\frac{\vartheta \operatorname{coth} \vartheta}{2\left(\delta x_{0}\right)^{2}}, \lambda^{2}=\frac{\vartheta \operatorname{coth} \vartheta}{2\left(\delta p_{0}\right)^{2}},
$$

where $\vartheta$ measures the size of the uncertainty product $\delta x_{0} \delta p_{0}$ as compared to its minimal value of $\hbar / 2$,

$$
\operatorname{coth} \vartheta \equiv \frac{2}{\hbar} \delta x_{0} \delta p_{0} \geq 1
$$

Consequently, the least-bias description of the situation specified by the knowledge of equations (3.1) employs the statistical operator

$$
\rho\left(t_{0}\right)=2 \sinh (\vartheta) \exp \left(-\frac{\vartheta}{2} \operatorname{coth}(\vartheta)\left[\left(\frac{x-x_{0}}{\delta x_{0}}\right)^{2}+\left(\frac{p-p_{0}}{\delta p_{0}}\right)^{2}\right]\right)
$$

The case of a minimum-uncertainty state, for which the equal sign holds in (3.6), is contained as the limit $\vartheta \rightarrow \infty$. The Wigner function of this $\rho$ is

$$
\rho_{\mathrm{w}}\left(t_{0}, x^{\prime}, p^{\prime}\right)=\frac{\hbar}{\delta x_{0} \delta p_{0}} \exp \left(-\frac{1}{2}\left(\frac{x^{\prime}-x_{0}}{\delta x_{0}}\right)^{2}-\frac{1}{2}\left(\frac{p^{\prime}-p_{0}}{\delta p_{0}}\right)^{2}\right)
$$

for which the $\delta x_{0} \delta p_{0}=\hbar / 2$ case is a simple matter.

It is instructive to consider the approximations (2.11) and (2.12) here. They read

$$
\begin{aligned}
Z_{\mathrm{w}}\left(x^{\prime}, p^{\prime}\right) & \cong \exp \left(-\kappa^{2}\left(x^{\prime}-a\right)^{2}-\lambda^{2}\left(p^{\prime}-b\right)^{2}\right) \\
\operatorname{tr}\{Z\} & \cong \frac{1}{2 \hbar \kappa \lambda}
\end{aligned}
$$

and produce, in conjunction with equations (3.3), the according approximations

$$
a \cong x_{0}, b \cong p_{0}, \kappa^{2} \cong \frac{1}{2\left(\delta x_{0}\right)^{2}}, \lambda^{2} \cong \frac{1}{2\left(\delta p_{0}\right)^{2}}
$$

Quite remarkably, the resulting approximation for $\rho_{\mathrm{w}}$ is identical with the exact answer (3.8). We thus observe that, in this example, the various approximations that are consequences of (2.11) compensate for each other in $\rho_{\mathrm{w}}$. We take this as additional encouragement for using (2.11) in more complicated situations, in which an exact answer is not available. 
3.2 KNown Velocity distribution. - Suppose that in addition to the position and its uncertainty at the initial time $t_{0}$, see equations (3.1a), one also knows the velocity distribution or probability density for momentum measurements,

$$
\left\langle p^{\prime}, t_{0}|\rho| p^{\prime}, t_{0}\right\rangle=\left\langle\delta\left(p\left(t_{0}\right)-p^{\prime}\right)\right\rangle=u\left(p^{\prime}\right) .
$$

Here, the ansatz

$$
\begin{aligned}
-\log Z & =\kappa^{2}(x-a)^{2}+\int \mathrm{d} p^{\prime} g\left(p^{\prime}\right) \delta\left(p-p^{\prime}\right) \\
& =\kappa^{2}(x-a)^{2}+g(p)
\end{aligned}
$$

is invited, where $x$ and $p$ refer to $t=t_{0}$. The values of the Lagrange multipliers $\kappa^{2}, a$, and $g\left(p^{\prime}\right)$ are fixed by $(3.3 \mathrm{a}),(3.3 \mathrm{c})$, and

$$
-\frac{\delta}{\delta g\left(p^{\prime}\right)} \log (\operatorname{tr}\{Z\})=u\left(p^{\prime}\right)
$$

For an arbitrary function $g\left(p^{\prime}\right)$, the trace of $Z$ cannot be evaluated explicitly. Therefore, we resort to the semiclassical approximations (2.11) and (2.12), which yield first

$$
a=x_{0}, \kappa^{2}=\frac{1}{2\left(\delta x_{0}\right)^{2}}, \frac{\exp \left(-g\left(p^{\prime}\right)\right)}{\int \mathrm{d} p^{\prime \prime} \exp \left(-g\left(p^{\prime \prime}\right)\right)}=u\left(p^{\prime}\right),
$$

and then

$$
\rho_{\mathrm{w}}\left(t_{0}, x^{\prime}, p^{\prime}\right)=\frac{\sqrt{2 \pi} \hbar}{\delta x_{0}} \exp \left(-\frac{1}{2}\left(\frac{x^{\prime}-x_{0}}{\delta x_{0}}\right)^{2}\right) u\left(p^{\prime}\right)
$$

The proper normalization of $\rho_{\mathbf{w}}$ is ensured by the property

$$
\int \mathrm{d} p^{\prime} u\left(p^{\prime}\right)=1
$$

necessarily possessed by the probability density $u\left(p^{\prime}\right)$.

The leading correction to the approximate result (3.15) is of the relative size [8]

$$
\left[\frac{1}{8}-\frac{1}{24}\left(\frac{x^{\prime}-x_{0}}{\delta x_{0}}\right)^{2}\right]\left(\frac{\hbar}{\delta x_{0}} \frac{\partial}{\partial p^{\prime}}\right)^{2} \log u\left(p^{\prime}\right)+\frac{1}{24}\left[\frac{\hbar}{\delta x_{0}} \frac{\partial}{\partial p^{\prime}} \log u\left(p^{\prime}\right)\right]^{2}
$$

As a demonstration of its extreme smallness, we compute it for a unidirectional thermal velocity distribution,

$$
u\left(p^{\prime}\right)=\left\{\begin{array}{cl}
0 & \text { for } p^{\prime}<0 \\
\frac{9}{2 p_{\mathrm{ml}}}\left(\frac{p^{\prime}}{p_{\mathrm{ml}}}\right)^{3} \exp \left(-\frac{3}{2}\left(\frac{p^{\prime}}{p_{\mathrm{ml}}}\right)^{2}\right) & \text { for } \quad p^{\prime}>0
\end{array}\right.
$$

where $p_{\mathrm{ml}}$ is the most likely momentum value. For this $u\left(p^{\prime}\right)$, the order of magnitude of the expression (3.17) is found to equal

$$
\left(\frac{\hbar}{\delta x_{0} p_{\mathrm{ml}}}\right)^{2}
$$


For the hydrogen beam in the experiment at Paris Nord [9], one has a velocity of $\sim 10^{6} \mathrm{~cm} / \mathrm{s}$, and the position where the metastable hydrogen is produced is known with a precision of about $5 \mathrm{~mm}$, so that

$$
\frac{\hbar}{\delta x_{0} p_{\mathrm{ml}}} \sim 10^{-9}
$$

and the relative size (3.17) of the leading correction to (3.15) is $\sim 10^{-18}-$ utterly negligible indeed.

3.3 PASSIVe Velocity SElection. - In the preceding example the initial velocity distribution was known, which is the situation in experiments with an active velocity selection, achieved, for instance, by a set of Fizeau wheels. Alternatively, one might opt for a passive velocity selection, realized by counting only those atoms that arrive in the detection region at $x=L \pm \delta L$ at a certain time $t=T$ chosen beforehand. Then the constraints (3.1a) at the initial time $t=t_{0}$ are supplemented by

$$
\langle x(T)\rangle=L,\left\langle(x(T))^{2}\right\rangle=L^{2}+(\delta L)^{2}
$$

Accordingly, the least-bias partition operator is of the form

$$
-\log Z=\kappa^{2}\left(x\left(t_{0}\right)-a\right)^{2}+\mu^{2}(x(T)-c)^{2}
$$

with $\kappa>0$ and $\mu>0$.

To proceed we need the dynamics to express $x(T)$ in terms of the operators $x\left(t_{0}\right)$ and $p\left(t_{0}\right)$ at the initial time $t_{0}$. For a spatially constant time-dependent force this is achieved with the aid of equations (2.17). Then (3.22) is unitarily equivalent to (3.2) provided that we set $\mu=m \lambda /\left(T-t_{0}\right)$ and $c=\left(T-t_{0}\right) b / m$. Accordingly, the trace of the statistical operator of (3.22) is given by (3.4) with $\lambda=\left(T-t_{0}\right) \mu / m$. This implies

$$
a=x_{0}, c=L, \kappa^{2}=\frac{\vartheta \operatorname{coth} \vartheta}{2\left(\delta x_{0}\right)^{2}}, \mu^{2}=\frac{\vartheta \operatorname{coth} \vartheta}{2(\delta L)^{2}},
$$

where

$$
\operatorname{coth} \vartheta=\frac{2}{\hbar} \frac{m}{T-t_{0}} \delta x_{0} \delta L \geq 1
$$

Note that the quantum mechanical "spreading of the wave function" sets a bound on $\delta x_{0} \delta L /(T-$ $\left.t_{0}\right)$.

Equation (2.18) supplies the statistical operator at the intermediate times. Its Wigner function, a two-dimensional Gaussian,

$$
\begin{array}{r}
\rho_{\mathrm{w}}\left(t, x^{\prime}, p^{\prime}\right)=\frac{T-t_{0}}{m} \frac{\hbar}{\delta x_{0} \delta L} \exp \left(-\frac{1}{2}\left(\frac{x^{\prime}-\left(t-t_{0}\right) p^{\prime} / m-s_{1}(t)}{\delta x_{0}}\right)^{2}\right) \\
\times \exp \left(-\frac{1}{2}\left(\frac{x^{\prime}+(T-t) p^{\prime} / m-s_{2}(t)}{\delta L}\right)^{2}\right)
\end{array}
$$

with

$$
\begin{aligned}
& s_{1}(t)=x_{0}-\int_{t_{0}}^{t} \mathrm{~d} t^{\prime}\left(t^{\prime}-t_{0}\right) F\left(t^{\prime}\right) / m, \\
& s_{2}(t)=L-\int_{t}^{T} \mathrm{~d} t^{\prime}\left(T-t^{\prime}\right) F\left(t^{\prime}\right) / m
\end{aligned}
$$


is a suitable generalization of (3.8). The physical significance of $s_{1}(t)$ and $s_{2}(t)$ is revealed by the expectation values of $x(t)$ and $p(t)$,

$$
\begin{aligned}
\langle x(t)\rangle & =\frac{T-t}{T-t_{0}} s_{1}(t)+\frac{t-t_{0}}{T-t_{0}} s_{2}(t), \\
\langle p(t)\rangle & =\frac{m}{T-t_{0}}\left(s_{2}(t)-s_{1}(t)\right) .
\end{aligned}
$$

One can use these relations to rewrite the Wigner function (3.25) in a more suggestive way,

$$
\begin{array}{r}
\rho_{\mathrm{w}}\left(t, x^{\prime}, p^{\prime}\right)=\frac{T-t_{0}}{m} \frac{\hbar}{\delta x_{0} \delta L} \exp \left(-\frac{1}{2}\left(\frac{\left[x^{\prime}-\langle x(t)\rangle\right]-\left(t-t_{0}\right)\left[p^{\prime}-\langle p(t)\rangle\right] / m}{\delta x_{0}}\right)^{2}\right) \\
\times \exp \left(-\frac{1}{2}\left(\frac{\left[x^{\prime}-\langle x(t)\rangle\right]+(T-t)\left[p^{\prime}-\langle p(t)\rangle\right] / m}{\delta L}\right)^{2}\right)
\end{array}
$$

We observe immediately that the maximum of $\rho_{\mathrm{w}}$ is located at $x^{\prime}=\langle x(t)\rangle, p^{\prime}=\langle p(t)\rangle$ where both Gaussian factors equal unity.

Not surprisingly, the expectation values (3.27) are of classical appearance. Quantum mechanics is, however, essential for the uncertainties,

$$
\begin{aligned}
\delta x(t) & =\sqrt{\left\langle x^{2}(t)\right\rangle-\langle x(t)\rangle^{2}}=\sqrt{\left(\frac{T-t}{T-t_{0}} \delta x_{0}\right)^{2}+\left(\frac{t-t_{0}}{T-t_{0}} \delta L\right)^{2}} \\
& \geq \frac{\delta x_{0} \delta L}{\sqrt{\left(\delta x_{0}\right)^{2}+(\delta L)^{2}}} \equiv \delta \bar{x} \\
\delta p & =\sqrt{\left\langle p^{2}(t)\right\rangle-\langle p(t)\rangle^{2}}=\frac{m}{T-t_{0}} \sqrt{\left(\delta x_{0}\right)^{2}+(\delta L)^{2}}
\end{aligned}
$$

and also for the difference

$$
\begin{aligned}
D(t) & \equiv \frac{1}{2}\langle(x(t) p(t)+p(t) x(t))\rangle-\langle x(t)\rangle\langle p(t)\rangle \\
& =-\frac{m}{\left(T-t_{0}\right)^{2}}\left[(T-t)\left(\delta x_{0}\right)^{2}-\left(t-t_{0}\right)(\delta L)^{2}\right]
\end{aligned}
$$

Please note that the right-hand sides of equations (3.29) do not involve the force $F(t)$. As a consequence, the general form of Heisenberg's uncertainty relation

$$
\sqrt{(\delta x \delta p)^{2}-D^{2}} \geq \frac{\hbar}{2}
$$

is here obeyed in a time-independent way. Indeed, it repeats the inequality of (3.24).

The momentum spread $\delta p$ in $(3.29 \mathrm{~b})$ is time independent. In contrast, from $t=t_{0}$ on, the position uncertainty decreases from $\delta x_{0}$ towards its minimal value $\delta \bar{x}$, which is reached for $t=\bar{t}$. This distinguished instant is a weighted average of $t_{0}$ and $T$,

$$
\bar{t}=\frac{\left(\delta x_{0}\right)^{2} T+(\delta L)^{2} t_{0}}{\left(\delta x_{0}\right)^{2}+(\delta L)^{2}}
$$

at this moment, the right-hand side of (3.29c) vanishes. After $t=\bar{t}, \delta x(t)$ increases until it equals $\delta L$ at $t=T$. The optimal "shrinking of the wave function" is obtained in the symmetrical situation $\delta x_{0}=\delta L$, when $\bar{t}=\left(T+t_{0}\right) / 2$ and $\delta \bar{x}=\delta x_{0} / \sqrt{2}$. 
With position dependent forces, matters can be quite different and much more complicated. Except for forces linear in $x$, an explicit solution of the equations of motion (2.16) is not available then. Depending on the circumstances, reasonable approximations could be provided for by linearizations, or by a selfconsistent solution of the semiclassical equations

$$
\frac{\mathrm{d}}{\mathrm{d} t} x(t)=p(t) / m, \frac{\mathrm{d}}{\mathrm{d} t} p(t)=F(t, x(t)) \cong F(t,\langle x(t)\rangle) \equiv F(t)
$$

which involve an effective time dependent force, or by a combination of both.

3.4 Time-OF-FLIGHT MEASUREMENTS. - A common way to determine the velocity distribution in a beam are time-of-flight measurements. The signal is proportional to the flux entering the detection region at $x=L$. If the velocity is given by $p(t) / m$, the probability current is

$$
\begin{aligned}
j\left(t, x^{\prime}\right) & =\left\langle x^{\prime}, t\left|\left(\frac{p(t)}{2 m} \rho(t)+\rho(t) \frac{p(t)}{2 m}\right)\right| x^{\prime}, t\right\rangle \\
& =\left\langle J\left(x(t)-x^{\prime}, p(t)\right)\right\rangle,
\end{aligned}
$$

with the current operator

$$
J\left(x-x^{\prime}, p\right)=\delta\left(x-x^{\prime}\right) \frac{p}{2 m}+\frac{p}{2 m} \delta\left(x-x^{\prime}\right) .
$$

The time-of-flight measurement supplies $j(t, L)$ as a function of the arrival time $t$. Here the constraints (3.1a) at the initial time $t_{0}$ are supplemented by

$$
\langle J(x(t)-L, p(t))\rangle=j(t, L)
$$

so that the ansatz

$$
-\log Z=\kappa^{2}\left(x\left(t_{0}\right)-a\right)^{2}+\int \mathrm{d} t f(t) J(x(t)-L, p(t))
$$

is appropriate for the least-bias partition operator.

Inasmuch as (3.36) involves operators at various times, the dynamics is needed, as in the preceding section, to relate them all to one common instant. The force-free motion is typical in the present context; it will serve us as the illustrating situation. We take the initial time $t_{0}$ for reference, so that

$$
-\log Z=\kappa^{2}(x-a)^{2}+\int \mathrm{d} t f(t) J\left(x+\frac{t-t_{0}}{m} p-L, p\right)
$$

with $x \equiv x\left(t_{0}\right)$ and $p \equiv p\left(t_{0}\right)$. The Wigner function of $\log Z$ is

$$
-(\log Z)_{\mathbf{w}}\left(x^{\prime}, p^{\prime}\right)=\kappa^{2}\left(x^{\prime}-a\right)^{2}+\frac{p^{\prime}}{\left|p^{\prime}\right|} f\left(t_{0}+\frac{m}{p^{\prime}}\left(L-x^{\prime}\right)\right)
$$

whose derivation uses

$$
\begin{aligned}
{\left[J\left(x+\frac{t-t_{0}}{m} p-L, p\right)\right]_{\mathrm{w}}\left(x^{\prime}, p^{\prime}\right) } & =\frac{p^{\prime}}{m} \delta\left(x^{\prime}+\frac{t-t_{0}}{m} p^{\prime}-L\right) \\
& =\frac{p^{\prime}}{\left|p^{\prime}\right|} \delta\left(t-t_{0}-\frac{m}{p^{\prime}}\left(L-x^{\prime}\right)\right) .
\end{aligned}
$$


In a reasonable experiment the condition $L-x_{0} \gg \delta x_{0}$ is obeyed and, since the relevant $x^{\prime}$ values are anticipated to be around $x_{0}$, only positive $p^{\prime}$ values have to be considered, so that the simplifying replacement $p^{\prime} /\left|p^{\prime}\right| \rightarrow 1$ is permissible. Then (2.12) yields

$$
\operatorname{tr}\{Z\}=\frac{m}{2 \sqrt{\pi} \hbar} \frac{L-a}{\kappa} \int \frac{\mathrm{d} \tau}{\tau^{2}} \exp \left(-f\left(t_{0}+\tau\right)\right) .
$$

Equations (3.3a) and (3.3c) imply

$$
\frac{1}{2 \kappa^{2}}=(L-a)\left(a-x_{0}\right)=\left(x_{0}-a\right)^{2}+\left(\delta x_{0}\right)^{2},
$$

and

$$
-\frac{\delta}{\delta f(t)} \log (\operatorname{tr}\{Z\})=j(t, L)
$$

produces the additional requirement

$$
\frac{\left(t-t_{0}\right)^{-2} \exp (-f(t))}{\int \mathrm{d} \tau \tau^{-2} \exp \left(-f\left(t_{0}+\tau\right)\right)}=\jmath(t, L)
$$

Consequently, we obtain

$$
\begin{aligned}
\rho_{\mathrm{w}}\left(t_{0}, x^{\prime}, p^{\prime}\right)= & 2 \sqrt{\pi} \hbar \frac{\kappa / m}{L-a} \exp \left(-\kappa^{2}\left(x^{\prime}-a\right)^{2}\right) \\
& \times\left.\tau^{2} j\left(t_{0}+\tau, L\right)\right|_{\tau=\frac{m}{p^{\prime}}\left(L-x^{\prime}\right)}
\end{aligned}
$$

with [10]

$$
\begin{aligned}
a & =x_{0}+\frac{L-x_{0}}{4}\left(1-\sqrt{1-8\left(\frac{\delta x_{0}}{L-x_{0}}\right)^{2}}\right) \\
\kappa^{2} & =\left[\left(\delta x_{0}\right)^{2}+\left(\frac{L-x_{0}}{2}\right)^{2}\left(1-\sqrt{1-8\left(\frac{\delta x_{0}}{L-x_{0}}\right)^{2}}\right)\right]^{-1}
\end{aligned}
$$

In view of $L-x_{0} \gg \delta x_{0}$, the Lagrange multipliers $a$ and $\kappa^{2}$ are very well approximated by

$$
a \cong x_{0}+\frac{\left(\delta x_{0}\right)^{2}}{L-x_{0}} \cong x_{0}, \kappa^{2} \cong \frac{1}{2\left(\delta x_{0}\right)^{2}}
$$

accordingly, (3.44) simplifies to

$$
\rho_{\mathrm{w}}\left(t_{0}, x^{\prime}, p^{\prime}\right)=\left.\frac{\sqrt{2 \pi} \hbar}{\delta x_{0}} \exp \left(-\frac{1}{2}\left(\frac{x^{\prime}-x_{0}}{\delta x_{0}}\right)^{2}\right) \frac{\tau^{2} / m}{L-x_{0}} j\left(t_{0}+\tau, L\right)\right|_{\tau=\frac{m}{p^{\prime}}}\left(L-x^{\prime}\right)
$$

The Gaussian $x^{\prime}$ dependence limits the relevant $x^{\prime}$ range to $x^{\prime}=x_{0} \pm \delta x_{0}$; therefore, the replacement

$$
\tau \rightarrow \frac{m}{p^{\prime}}\left(L-x_{0}\right) \equiv \tau_{p^{\prime}}
$$


discards terms that are of relative size $\left(\frac{\delta x_{0}}{L-x_{0}}\right)^{2} \ll 1$. Then (3.47) is identical with (3.15), whereby

$$
p^{\prime} u\left(p^{\prime}\right)=\tau_{p^{\prime}} \jmath\left(t_{0}+\tau_{p^{\prime}}, L\right),
$$

relates the velocity distribution $u\left(p^{\prime}\right)$ and the time-of-flight signal $j\left(t_{0}+\tau_{p^{\prime}}, L\right)$ to each other. These relations are, of course, what would emerge also from classical kinematics. If desired, the very small quantal corrections can be computed by inserting the Wigner function of (3.44) into the general expression

$$
u\left(p^{\prime}\right)=\int \frac{\mathrm{d} x^{\prime}}{2 \pi \hbar} \rho_{\mathrm{w}}\left(x^{\prime}, p^{\prime}\right)
$$

The data of the time-of-flight measurements on a hydrogen beam performed in Villetaneuse are consistent with [9]

$$
j\left(t_{0}+\tau, L\right)=\frac{25}{2 \tau_{\mathrm{ml}}}\left(\frac{\tau_{\mathrm{ml}}}{\tau}\right)^{5} \exp \left(-\frac{5}{2}\left(\frac{\tau_{\mathrm{ml}}}{\tau}\right)^{2}\right) \text { for } \tau>0,
$$

where $\tau_{\mathrm{ml}}$ denotes the most likely delay. The velocity distribution thus implied by (3.49) is of the thermal form (3.18) with $p_{\mathrm{ml}} \tau_{\mathrm{ml}}=\sqrt{3 / 5} m\left(L-x_{0}\right)$.

\section{An application to atomic interferometry.}

In the recent experiment reported in reference [11] magnetic atoms move through a set of Helmholtz coils. A passive velocity selection, as discussed in section 3.3, chooses those atoms that are, during a known time interval, in the spatial region where the magnetic field is homogeneous. When the atoms are there, a current is sent through the Helmholtz coils. As a result, the atoms are exposed to a spatially constant magnetic field of varying strength for a certain time of adjustable duration. The scalar Aharanov-Bohm effect, and in particular its non-dispersivity, are demonstrated in this way.

In a variant of this experiment one could employ a different geometry of the magnetic field, in which one has a relatively long stretch where the field gradient is spatially constant. When the current is switched on for a judiciously chosen time interval, all atoms in the passively-selected velocity range are exposed to a spatially constant force that depends on the internal spin state. Consequently, the various spin components of the atomic state are affected differently and the final spin state will depend on the strength and duration of the current applied.

Although metastable hydrogen atoms in a spin-1 state are used in the experiments of [9] and [11], we discuss the somewhat simpler case of spin- $\frac{1}{2}$ atoms. Near the $x$ axis, along which the beam propagates, the magnetic field is in the $z$ direction and its strength varies linearly with $x$ in the region of interest. We ignore the CM motion in the $y, z$ plane and model, in the spirit of equations (3.32), the dynamics with the aid of the Hamilton operator

$$
H=\frac{p^{2}}{2 m}+\frac{\hbar}{2} \Omega_{0}(t) \sigma_{z}-F(t) x \sigma_{z}
$$

where $\Omega_{0}(t)$, the Larmor frequency at $x=0$, and the force $F(t)$ are explicitly time dependent. As usual, the spin- $\frac{1}{2}$ degree of freedom is described by Pauli operators $\sigma_{z}$ and $\sigma_{ \pm}=\sigma_{x} \pm \imath \sigma_{y}$, which are sometimes conveniently comprised into the vector operator $\sigma$.

The force-free situation, when $F(t) \equiv 0$, is contained in (4.1) as a special case. Then the time dependence of the Larmor frequency gives rise to the scalar Aharanov-Bohm effect. A recent experimental study is reported in reference [11]. 
The equations of motion implied by the Hamilton operator (4.1),

$$
\begin{aligned}
\frac{\mathrm{d}}{\mathrm{d} t} x(t) & =p(t) / m, \\
\frac{\mathrm{d}}{\mathrm{d} t} p(t) & =F(t) \sigma_{z}(t), \\
\frac{\mathrm{d}}{\mathrm{d} t} \sigma_{z}(t) & =0 \\
\frac{\mathrm{d}}{\mathrm{d} t} \sigma_{ \pm}(t) & = \pm i\left[\Omega_{0}(t)-\frac{2}{\hbar} F(t) x(t)\right] \sigma_{ \pm}(t),
\end{aligned}
$$

are solved by [12]

$$
\begin{aligned}
x(t) & =x+p \frac{t-t_{0}}{m}+\sigma_{z} s(t) \\
p(t) & =p+\sigma_{z} q(t) \\
\sigma_{z}(t) & =\sigma_{z} \\
\sigma_{ \pm}(t) & =\exp \left( \pm i \phi_{0}(t) \mp \frac{2 i}{\hbar}[x q(t)+p S(t)]\right) \sigma_{ \pm},
\end{aligned}
$$

where the operators $x, p, \sigma_{z}, \sigma_{ \pm}$on the right-hand sides refer to the initial time $t=t_{0}$. The numerical functions $s(t), q(t)$, and $S(t)$ are integrals over the force $F(t)$,

$$
\begin{aligned}
s(t) & =\int_{t_{0}}^{t} \mathrm{~d} t^{\prime} \frac{t-t^{\prime}}{m} F\left(t^{\prime}\right), \\
q(t) & =\int_{t_{0}}^{t} \mathrm{~d} t^{\prime} F\left(t^{\prime}\right)=m \frac{\partial}{\partial t} s(t), \\
S(t) & =\int_{t_{0}}^{t} \mathrm{~d} t^{\prime} \frac{t^{\prime}-t_{0}}{m} F\left(t^{\prime}\right)=\frac{t-t_{0}}{m} q(t)-s(t),
\end{aligned}
$$

and

$$
\phi_{0}(t)=\int_{t_{0}}^{t} \mathrm{~d} t^{\prime} \Omega_{0}\left(t^{\prime}\right)
$$

is the Larmor precession angle that would be accumulated at $x=0$.

In addition to (3.1a) we require

$$
\left\langle\sigma_{x}\left(t_{0}\right)\right\rangle=1
$$

initially. At the final time $T$, equations (3.21) have to hold. Consistency demands that all atoms thus selected are in the constant-gradient region while the current is switched on; otherwise the Hamilton operator (4.1) would not apply. Formally we have

$$
F(t)=0 \text { for }\left\{\begin{array}{c}
t_{0}<t<t_{1} \\
\text { and } \\
t_{2}<t<T
\end{array}\right.
$$

where $t_{1} \cdot t_{2}$ is the time interval during which the field gradient is present. If the corresponding $x$ interval is $x_{1} \cdot x_{2}$, then we must have

$$
\begin{aligned}
x_{0}<x_{1}< & \langle x(t)\rangle_{ \pm}-\delta x(t)_{ \pm} \\
& \langle x(t)\rangle_{ \pm}+\delta x(t)_{ \pm} \quad<x_{2}<L
\end{aligned}
$$


for $t_{1}<t<t_{2}$. The notation $\langle\cdot \cdot\rangle_{ \pm}$is short-hand for the expectation values of the natural spin components,

$$
\langle x(t)\rangle_{ \pm} \equiv \frac{\left\langle\frac{1}{2}\left[1 \pm \sigma_{z}(t)\right] x(t)\right\rangle}{\left\langle\frac{1}{2}\left[1 \pm \sigma_{z}(t)\right]\right\rangle}=\left\langle\left[1 \pm \sigma_{z}(t)\right] x(t)\right\rangle=\operatorname{tr}_{\circ} \operatorname{tr}_{\sigma}\left\{\left[1 \pm \sigma_{z}(t)\right] x(t) \rho(t)\right\}
$$

which makes use of $\left\langle\sigma_{z}(t)\right\rangle=\left\langle\sigma_{z}\left(t_{0}\right)\right\rangle=0$, as implied by (4.3) and (4.6). The statistical operator $\rho(t)=\rho(t, x(t), p(t), \sigma(t))$ here depends both on the CM variables $x, p$ (the subscript ' $O$ ' stands for orbital) and on the spin vector $\sigma$.

The uncertainties $\delta x(t)_{ \pm}$are both given by (3.29a) because there the force does not enter. In contrast, the global spread

$$
\delta x(t)=\sqrt{\left\langle x^{2}(t)\right\rangle-\langle x(t)\rangle^{2}}=\sqrt{\frac{1}{2}\left[\left\langle x^{2}\right\rangle_{+}+\left\langle x^{2}\right\rangle_{-}\right]-\frac{1}{4}\left[\langle x\rangle_{+}+\langle x\rangle_{-}\right]^{2}}
$$

has to account for the longitudinal splitting of the beam and must, therefore, involve the force $F(t)$. Indeed, it does, as is seen in

$$
\delta x(t)=\sqrt{\left(\frac{T-t}{T-t_{0}} \delta x_{0}\right)^{2}+\left(\frac{t-t_{0}}{T-t_{0}} \delta L\right)^{2}+\frac{1}{4}\left[\langle x(t)\rangle_{+}-\langle x(t)\rangle_{-}\right]^{2}} .
$$

We note that the requirements (4.8) are more stringent than the global ones,

$$
\begin{aligned}
x_{1}< & \langle x(t)\rangle-\delta x(t), \\
& \langle x(t)\rangle+\delta x(t) \quad<x_{2},
\end{aligned}
$$

would be.

As compared to (3.22), the appropriate least-bias partition operator

$$
-\log Z=\kappa^{2}\left(x\left(t_{0}\right)-a\right)^{2}+\mu^{2}(x(T)-c)^{2}+\alpha \sigma_{x}\left(t_{0}\right)
$$

has an extra term that incorporates (4.6). Again we express $x(T)$ in terms of the operators at the initial time $t_{0}$, for which purpose (4.3) is used, and arrive at

$$
-\log Z=U(-\log \tilde{Z}) U^{-1}=-\log \left(U \tilde{Z} U^{-1}\right)
$$

where

$$
\begin{aligned}
-\log \tilde{Z}= & \kappa^{2}(x-a)^{2}+\mu^{2}\left(x+p \frac{T-t_{0}}{m}-c\right)^{2} \\
& +\alpha\left[\sigma_{x} \cos \left(\frac{2}{\hbar} \frac{m}{T-t_{0}} s(T) x\right)-\sigma_{y} \sin \left(\frac{2}{\hbar} \frac{m}{T-t_{0}} s(T) x\right)\right]
\end{aligned}
$$

is unitarily equivalent to $-\log Z$. The unitary operator in $(4.14)$ is

$$
U=\exp \left(-\frac{i}{\hbar} \frac{m}{T-t_{0}} s(T) x \sigma_{z}\right) .
$$

It effects the transformations

$$
\begin{aligned}
U^{-1} p U & =p-\frac{m}{T-t_{0}} s(T) \sigma_{z} \\
U^{-1} \sigma_{ \pm} U & =\exp \left( \pm \frac{2 i}{\hbar} \frac{m}{T-t_{0}} s(T) x\right) \sigma_{ \pm}
\end{aligned}
$$


and commutes with $x$ and $\sigma_{z}$. When using

$$
\begin{aligned}
\exp \left((\log \tilde{Z})_{\mathrm{w}}\right. & \left.\left(x^{\prime}, p^{\prime}\right)\right)=\exp \left(-\kappa^{2}\left(x^{\prime}-a\right)^{2}-\mu^{2}\left(x^{\prime}+p^{\prime} \frac{T-t_{0}}{m}-c\right)^{2}\right) \\
\times & {\left[\cosh \alpha-\left(\sigma_{x} \cos \left(\frac{2}{\hbar} \frac{m}{T-t_{0}} s(T) x^{\prime}\right)-\sigma_{y} \sin \left(\frac{2}{\hbar} \frac{m}{T-t_{0}} s(T) x^{\prime}\right)\right) \sinh \alpha\right] }
\end{aligned}
$$

as an approximation to $\tilde{Z}_{\mathrm{w}}\left(x^{\prime}, p^{\prime}\right)$ we find

$$
\operatorname{Tr}\{Z\}=\operatorname{Tr}\{\tilde{Z}\}=\int \frac{\mathrm{d} x^{\prime} \mathrm{d} p^{\prime}}{2 \pi \hbar} \operatorname{tr}_{\sigma}\left\{\tilde{Z}_{\mathbf{w}}\left(x^{\prime}, p^{\prime}\right)\right\} \cong \frac{m}{T-t_{0}} \frac{\cosh \alpha}{\hbar \kappa \mu}
$$

where $\operatorname{Tr} \equiv \operatorname{tr}_{\mathrm{o}} \operatorname{tr}_{\sigma}$ is the total trace. The constraints (3.1a), (3.21), and (4.6) then imply

$$
a=x_{0}, c=L, \kappa^{2}=\frac{1}{2\left(\delta x_{0}\right)^{2}}, \mu^{2}=\frac{1}{2(\delta L)^{2}}, \tanh \alpha=-1,
$$

so that (4.18) and (4.19) produce

$$
\begin{aligned}
\tilde{\rho}_{\mathrm{w}}\left(t_{0}, x^{\prime}, p^{\prime}\right)= & \frac{T-t_{0}}{m} \frac{\hbar}{\delta x_{0} \delta L} \exp \left(-\frac{1}{2}\left(\frac{x^{\prime}-x_{0}}{\delta x_{0}}\right)^{2}-\frac{1}{2}\left(\frac{x^{\prime}+\left(T-t_{0}\right) p^{\prime} / m-L}{\delta L}\right)^{2}\right) \\
& \times \frac{1}{2}\left[1+\sigma_{x} \cos \left(\frac{2}{\hbar} \frac{m}{T-t_{0}} s(T) x^{\prime}\right)-\sigma_{y} \sin \left(\frac{2}{\hbar} \frac{m}{T-t_{0}} s(T) x^{\prime}\right)\right] .
\end{aligned}
$$

In accordance with (4.14) we have $\rho=U \tilde{\rho} U^{-1}$, with the consequence that the expectation value of an observable $\mathcal{O}\left(x, p ; \sigma_{z}, \sigma_{+}, \sigma_{-}\right)$is given by

$$
\langle\mathcal{O}\rangle=\operatorname{Tr}\{\mathcal{O} \rho\}=\operatorname{Tr}\left\{U^{-1} \mathcal{O} U \tilde{\rho}\right\}
$$

where

$$
U^{-1} \mathcal{O}\left(x, p ; \sigma_{z}, \sigma_{+}, \sigma_{-}\right) U=\mathcal{O}\left(x, U^{-1} p U ; \sigma_{z}, U^{-1} \sigma_{+} U, U^{-1} \sigma_{-} U\right)
$$

involves the transformations (4.17). In conjunction with $\tilde{\rho}_{\mathbf{w}}$ of (4.21), the Wigner function of $U^{-1} \mathcal{O} U$ is employed for an (approximate) evaluation of $\langle\mathcal{O}\rangle$,

$$
\langle\mathcal{O}\rangle=\int \frac{\mathrm{d} x^{\prime} \mathrm{d} p^{\prime}}{2 \pi \hbar} \operatorname{tr}_{\sigma}\left\{\left(U^{-1} \mathcal{O} U\right)_{\mathrm{w}} \tilde{\rho}_{\mathrm{w}}\right\}
$$

in terms of a phase space integral.

The solutions (4.3) of the equations of motion (4.2) together with the transformations (4.17) supply the Wigner functions

$$
\begin{aligned}
& {\left[U^{-1}\left(1 \pm \sigma_{z}\right) x(t) U\right]_{\mathrm{w}}\left(x^{\prime}, p^{\prime}\right)=\left(1 \pm \sigma_{z}\right)\left[\left(x^{\prime}+\frac{t-t_{0}}{m} p^{\prime}\right) \pm\left(s(t)-\frac{t-t_{0}}{T-t_{0}} s(T)\right)\right]} \\
& {\left[U^{-1}\left(1 \pm \sigma_{z}\right) p(t) U\right]_{\mathrm{w}}\left(x^{\prime}, p^{\prime}\right)=\left(1 \pm \sigma_{z}\right)\left[p^{\prime} \pm\left(q(t)-\frac{m}{T-t_{0}} s(T)\right)\right]}
\end{aligned}
$$

as well as

$$
\left[U^{-1} \sigma_{ \pm}(t) U\right]_{\mathrm{w}}\left(x^{\prime}, p^{\prime}\right)=\sigma_{ \pm} \exp \left( \pm i \phi_{0}(t) \mp \frac{2 i}{\hbar} p^{\prime} S(t) \mp \frac{2 i}{\hbar} x^{\prime}\left(q(t)-\frac{m}{T-t_{0}} s(T)\right)\right) .
$$


The mean positions of the spin components thus obtained are

$$
\langle x(t)\rangle_{ \pm}=\frac{T-t}{T-t_{0}} x_{0}+\frac{t-t_{0}}{T-t_{0}} L \mp \int_{t_{0}}^{T} \mathrm{~d} t^{\prime} \frac{\left(T-t_{>}\right)\left(t_{<}-t_{0}\right)}{T-t_{0}} \frac{F\left(t^{\prime}\right)}{m},
$$

where $t_{>}$denotes the later one of the two times $t$ and $t^{\prime}$, and $t_{<}$the earlier one. These expectation values are needed in the consistency check (4.8). The corresponding average momenta are

$$
\langle p(t)\rangle_{ \pm}=\frac{m}{T-t_{0}}\left(L-x_{0}\right) \pm\left(q(t)-\frac{m}{T-t_{0}} s(T)\right) .
$$

Of course, $\langle x\rangle_{+}$and $\langle p\rangle_{+}$agree with the expectation values (3.27). By construction, the locations of both spin components are the same at the initial and at the final time. Since opposite forces act on the spin components, their momenta must differ, however. In other words, the internal spin degree of freedom is entangled with the external CM motion. In particular, we have initially

$$
\Delta p\left(t_{0}\right)=\left\langle p\left(t_{0}\right)\right\rangle_{+}-\left\langle p\left(t_{0}\right)\right\rangle_{-}=-\frac{2 m}{T-t_{0}} s(T)=-2 \int_{t_{0}}^{T} \mathrm{~d} t^{\prime} \frac{T-t^{\prime}}{T-t_{0}} F\left(t^{\prime}\right)
$$

and finally

$$
\Delta p(T)=\langle p(T)\rangle_{+}-\langle p(T)\rangle_{-}=\frac{2 m}{T-t_{0}} S(T)=2 \int_{t_{0}}^{T} \mathrm{~d} t^{\prime} \frac{t^{\prime}-t_{0}}{T-t_{0}} F\left(t^{\prime}\right) .
$$

Please note that the passive velocity selection picks different momentum ranges for the two spin components, viz.

$$
\left\langle p\left(t_{0}\right)\right\rangle \pm \frac{1}{2} \Delta p\left(t_{0}\right)-\delta p_{ \pm} \lesssim p \lesssim\left\langle p\left(t_{0}\right)\right\rangle \pm \frac{1}{2} \Delta p\left(t_{0}\right)+\delta p_{ \pm}
$$

for $\sigma_{z}= \pm 1$, where $\langle p\rangle=\left(\langle p\rangle_{+}+\langle p\rangle_{-}\right) / 2$ and the spreads $\delta p_{ \pm}$are both given by (3.29b). These differing ranges are necessary to ensure that all atoms move form $x=x_{0}$ at $t=t_{0}$ to $x=L$ at $t=T$ although a spin dependent force is acting at intermediate times.

Further, concerning the time dependence of the atom's polarization, we find

$$
\left\langle\sigma_{ \pm}(t)\right\rangle=\exp \left( \pm i \phi(t)-2\left(\frac{m \delta L / \hbar}{T-t_{0}} S(t)\right)^{2}-2\left(\frac{m s(t)+(T-t) q(t)}{T-t_{0}} \frac{\delta x_{0}}{\hbar}\right)^{2}\right)
$$

where

$$
\phi(t)=\int_{t_{0}}^{t} \mathrm{~d} t^{\prime}\left[\Omega_{0}\left(t^{\prime}\right)-\frac{2}{\hbar} F\left(t^{\prime}\right)\left\langle x\left(t^{\prime}\right)\right\rangle\right]
$$

is the total Larmor precession angle accumulated along the trajectory defined by the central position $\langle x(t)\rangle=\left(\langle x(t)\rangle_{+}+\langle x(t)\rangle_{-}\right) / 2$. At the final time $T$, when the orientation of the spin vector is actually detected, we have the transparent result

$$
\left\langle\sigma_{ \pm}(T)\right\rangle=\exp ( \pm \imath \phi(T)) \exp \left(-\frac{1}{2}\left(\frac{\Delta p\left(t_{0}\right) \delta x_{0}}{\hbar}\right)^{2}\right) \exp \left(-\frac{1}{2}\left(\frac{\Delta p(T) \delta L}{\hbar}\right)^{2}\right)
$$

The quantity $C \equiv|\langle\boldsymbol{\sigma}\rangle|=\left[\left\langle\sigma_{z}\right\rangle^{2}+\left\langle\sigma_{+}\right\rangle\left\langle\sigma_{-}\right\rangle\right]^{1 / 2}$ is the numerical measure for the spin coherence [13]; its final value $C(T)$ is given by the product of the two Gaussians in (4.33). This number 
sets an upper bound on the contrast of the interference pattern - in a measurement of $\langle(1+$ $\left.\left.\sigma_{x}\right) / 2\right\rangle$, say. A reasonable visibility of the fringes requires therefore that

$$
\left|\Delta p\left(t_{0}\right)\right| \lesssim \frac{\hbar}{\delta x_{0}} \text { and }|\Delta p(T)| \lesssim \frac{\hbar}{\delta L}
$$

hold. We conclude: when the force $F(t)$ gets too strong, the fringes disappear.

In the force-free situation of the scalar Aharanov-Bohm effect one has $\Delta p\left(t_{0}\right)=0$ and $\Delta p(T)=0$ in (4.33). Then a contrast of $100 \%$ is conceivable, at least as far as the kinematical effects of the CM motion are concerned. A real experiment [11] has other limitations in addition.

\section{Summary.}

We have introduced the concept of a least-bias statistical operator that correctly represents, without any hidden assumptions, what is known about the CM degree of freedom of those atoms in a beam that contribute to the signal in a given experimental situation. In particular, the popular bias that the CM motion should be describable by a pure state is avoided. We have demonstrated the method by explicit treatments of a few exemplary situations which bear relevance for actual experiments. We have found that the standard way of inferring the velocity distribution from time-of-flight measurements, which is based upon classical kinematics, is well-justified because the quantum corrections are negligible for reasonable experimental parameters. Finally, we have employed the formalism for a theoretical study of magnetic atoms that experience a pulsed field gradient in a longitudinal Stern-Gerlach interferometer. We have found the dependence of the fringe visibility upon the strength and duration of the pulse.

\section{Acknowledgments.}

B.-G. E. would like to express his gratitude to Martial Ducloy for the generous hospitality experienced in Villetaneuse. We thank S. Nic Chormaic, O. Gorceix, F. Perales, and J. Robert for stimulating discussions.

\section{References}

[1] A review is given by Hillery M., O'Connell R.F., Scully M.O. and Wigner E. P., Phys. Rep. 106 (1984) 121;

We use the normalization and conventions of B.-G. Englert, J. Phys. A22 (1989) 625.

[2] For background in statistical mechanics the reader might consult Balian R., From Microphysics to Macrophysics (Springer-Verlag, Berlin, 1991).

[3] Wigner E., Phys. Rev. 40 (1932) 749.

[4] Rohwedder B. and Englert B.-G., Phys. Rev. A 49 (1994) 2340.

[5] We note that equation (2.11) of the present paper is a special case of equation (2.5) in reference [4] or of equation (A1) in reference [8].

[6] Englert B.-G., New Frontiers in Quantum Electrodynamics and Quantum Optics, A.O. Barut Ed. (Plenum Press, New York, 1990).

[7] Zaugg T., Meystre P., Lenz G. and Wilkens M., Phys. Rev. A 49 (1994) 3011.

[8] See, for instance, equation (A4) in Cinal M. and Englert B.-G., Phys. Rev. A 48 (1993) 1893. 
[9] Robert J., Miniatura C., Gorceix O., Le Boiteux S., Lorent V., Reinhardt J. and Baudon J., J. Phys. II France 2 (1992) 601.

[10] The second root of the quadratic equation (3.41) that determines $a$ is discarded because the resulting density operator has a lower entropy. In addition, this second root is not acceptable since it would not approach $x_{0}$ in the limit $\left(L-x_{0}\right) / \delta x_{0} \rightarrow \infty$.

[11] Nic Chormaic S., Miniatura C., Gorceix O., Viaris de Lesegno B., Robert J., Feron S., Lorent V., Reinhardt J., Baudon J. and Rubin K., Phys. Rev. Lett. 72 (1994) 1.

[12] Englert B.-G., J. Schwinger J. and Scully M.O., Found. Phys. 18 (1988) 1045.

[13] Schwinger J., Scully M.O. and Englert B.-G., Z. Phys. 10 (1988) 135. 